\section{MS5-P12 Structural characterization of the mycobacterial ATP synthase c-ring and its interaction with the novel anti-tuberculosis drug Bedaquiline}

Laura Preiss ${ }^{1}$, Bianca Eisel ${ }^{1}$, Ernst Grell $^{2}$, Luise Eckhardt-Strelau ${ }^{1}$, Özkan Yildiz', Anil Koul ${ }^{3}$, Thomas K. Meier $^{1,4}$

1. Max-Planck-Institute of Biophysics, Department of Structural Biology, Frankfurt, Germany

2. Max-Planck-Institute of Biophysics, Department of Molecular Membrane Biology, Frankfurt, Germany

3. Janssen R\&D (Johnson\&Johnson Pharmaceuticals), Beerse, Belgium

4. Imperial College London, Department of Life Sciences, London, UK

email: laura.preiss@biophys.mpg.de

Tuberculosis (TB) disease is a world-wide health problem being responsible for more than 1.5 million deaths in 2014 (WHO, 2016) with the tendency to increase due to rapidly rising numbers of drug resistant Mycobacterium tuberculosis strains. New antibiotics are therefore urgently required. The novel, recently FDA approved antibiotic bedaquiline (BDQ) efficiently kills drug-sensitive and drug-resistant $M$. tuberculosis cells in latent and active infections. Initial genetic and biochemical studies suggested the bacterium's $\mathrm{F}_{1} \mathrm{~F}_{\mathrm{o}}$ ATP-synthase rotor (c-) ring as the drug's target (Andries et al., 2005; Haagsma et al., 2010; Koul et al., 2007). To gain further insights into the drug-target interaction and the molecular basis for the drug's high specificity, we solved the high-resolution X-ray structures of the c-ring from the non-pathogenic Mycobacterium phlei with and without BDQ at $1.7 \AA$ and $1.5 \AA$, respectively (Preiss et al., 2015). In agreement with our biochemical data, BDQ was bound to the c-ring's proton binding sites deeply buried in the middle of the membrane. The tight drug-target interaction network is composed of numerous van der Waals interactions and essential hydrogen bonds with the mycobacterial c-ring's surface, which is uniquely shaped by the conserved amino acid sequence of the mycobacterial c-subunit. Furthermore we performed a biochemical study with different purified $M$. phlei c-ring mutants to quantitatively evaluate the energetic contributions of each interaction using isothermal titration calorimetry (ITC). Some of the investigated mutants still showed a wild type-like binding behavior, while others had a significantly reduced affinity towards BDQ. Thus, our study revealed interactions that are essential for high affinity BDQ binding and mutations that are likely to cause BDQ resistance. These results give new insights into the ATP synthase c-ring-BDQ interaction and provide a rational basis for chemical adaptation and optimization of the novel anti-TB drug BDQ.

Keywords: ATP-synthase, tuberculosis disease, c-ring, bedaquiline, drug-target interaction
MS5-P13 Neutron crystallographic studies of cancer-related human carbonic anhydrase IX reveal details of hydrogen-bonding in inhibitor binding.

\author{
Zoë Fisher ${ }^{1}$, Katarina Koruza ${ }^{2}$, Brian $\mathrm{Mahon}^{3}, \mathrm{Cynthia} \mathrm{Okoh}^{3}$,
} Wolfgang Knecht ${ }^{4}$, Robert McKenna ${ }^{3}$

1. Scientific Activities Division, European Spallation Source, Lund, Sweden

2. Biology Department, Lund University, Lund, Sweden

3. Department of Biochemistry and Molecular Biology, University of Florida, Gainesville FL, USA

4. Biology Department, Lund Protein Production Platform, Lund University, Lund, Sweden

email: zoe.fisher@esss.se

Human carbonic anhydrase IX (HCA IX) expression in aggressive tumours, under hypoxic conditions, is an indicator of metastasis and poor cancer patient prognosis. As such, HCA IX has emerged as an important cancer imaging, diagnostic, and therapeutic target. Medicinal efforts to develop specific inhibitors for HCA IX are complicated by the presence of 14 other HCA isoforms that share both a common structural fold and exhibit high sequence similarity. It has been well established that ligand (inhibitor) binding to a target protein is mediated through numerous interactions that may include: H-bonding directly and/or through intervening waters, electrostatic interactions with charged or polar amino acid side chains, metal coordination, energetic changes through water displacement, aromatic stacking, or other hydrophobic interactions. As the magnitude of X-ray scattering from an atom depends on the atomic number (Z) for that element, the light atoms in protein crystals contribute very little, if at all, to X-ray scattering data, making the observation of hydrogen atoms almost impossible. Neutron diffraction offers a highly complementary approach in that the neutrons are scattered from atomic nuclei of all elements to a similar extent. In practice this means that the nuclear density maps for $\mathrm{C}, \mathrm{N}, \mathrm{D}$ (the isotope of $\mathrm{H}$ ), and $\mathrm{O}$ atoms all appear to a similar extent, even at medium ( 2 $\AA$ ) resolution. In this way neutron crystallography offers a powerful approach to observe the details of ligand binding that involves $\mathrm{H}$ atoms. Our goal is to determine the atomic details of new promising drug leads, complexed to HCA IX, by neutron protein crystallography. Saccharin was recently identified as a promising lead compound in that it demonstrates HCA IX specificity. Comparing neutron crystal structures of unbound and inhibitor-bound HCA IX provides a unique opportunity to directly investigate how saccharin binds through H-bonding, the role of water displacement, and how the making/breaking of H-bonds modulates binding and isoform specificity. It is expected this fine structural detail, that is unique to neutron crystallography, can then be applied for rational drug design.

Keywords: hydrogen bond, neutron crystallography, carbonic anhydrase 